\title{
Structural Analysis of Porphyrin Multilayer Films on ITO Assembled Using Copper(I)-Catalyzed Azide-Alkyne Cycloaddition by ATR IR
}

\author{
Peter K. B. Palomaki and Peter H. Dinolfo* \\ Department of Chemistry and Chemical Biology and The Baruch ' 60 Center for Biochemical Solar Energy Research, \\ Rensselaer Polytechnic Institute, 110 8th Street, Troy, New York 12180, United States
}

Supporting Information

ABSTRACT: We report the use of grazing-angle attenuated total reflectance (GATR) IR and polarized UV-vis to determine the molecular structure of porphyrin based molecular multilayer films grown in a layer-by-layer (LbL) fashion using coppercatalyzed azide-alkyne cycloaddition (CuAAC). The molecular orientation and bonding motif present in multilayer films of this type could impact their photophysical and electrochemical properties as well as potential applications. Multilayer films of $\mathrm{M}$ (II) 5,10,15,20-tetra(4-ethynylphenyl)porphyrin (1 M = Zn,

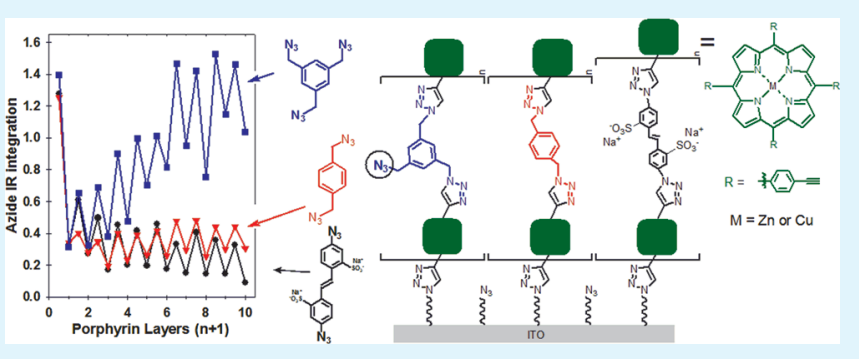
$2 \mathrm{M}=\mathrm{Cu}$ ) and azido based linkers 3-5 were used to fabricate the films on ITO substrates. Electrochemically determined coverage of films containing 2 match the trends observed in the absorbance. GATR-IR spectral analysis of the films indicate that CuAAC reactivity is leading to 1,4-triazole linked multilayers with increasing porphyrin and linker IR characteristic peaks. Films grown using all azido-linkers (3-5) display an oscillating trend in azide IR intensity suggesting that the surface bound azido group reacts with $\mathbf{1}$ and that further layering can occur through additional reaction with linkers, regenerating the azide surface. Films containing linker $\mathbf{5}$ in particular show an overall increase in azide content suggesting that only two of the three available groups react during multilayer fabrication, causing an overall buildup of azide content in the film. Films of 1 with linker 3 and $\mathbf{5}$ showed an average porphyrin plane angle of $46.4^{\circ}$ with respect to the substrate as determined by GATR FT-IR. Polarized UV-vis absorbance measurements correlate well with the growth angle calculated by IR. The orientation of the porphyrin plane within the multilayer structures suggests that the CuAAC-LbL process results in a film with a trans bonding motif.

KEYWORDS: layer-by-layer, thin-films, CuAAC, GATR-IR, porphyrin, multilayers

\section{INTRODUCTION}

Thin film materials fabricated in a layer-by-layer ( $\mathrm{LbL}$ ) fashion boast several advantages over other deposition techniques including ease of fabrication, rapid deposition, and multilayer films that are ordered in at least one direction. $\mathrm{LbL}$ assembled films, by design, often result in materials with ordering on the nano- to micrometer scale and properties based on the component building blocks. Thin film materials with nanoscale molecular ordering have implications in the areas of molecular electronics, photovoltaics, sensors, nonlinear optics, and more. ${ }^{1,2}$ Molecular LbL thin film fabrication (also known as molecular layer deposition, MLD) is a bottom-up approach that allows for the creation of materials with such nanoscale control. The process allows one to tailor film properties with respect to electronic, photophysical, and macroscopic properties, which could be useful towards the various applications listed above. Several chemical coupling reactions have been used to fabricate molecular-based multilayer films including zirconium phosphate linkages, ${ }^{2-5}$ palladium-pyridine coordination, ${ }^{6-10}$ siloxane polymerization, ${ }^{2,11-15}$ as well as other organic reactions. ${ }^{1,16-21}$ These types of reactions have resulted in well-ordered films that can incorporate various molecular building blocks. Molecular order within LbL films can result in films with interesting electrochemical and photophysical properties. $1,2,22$

We have recently developed a method to fabricate molecular multilayer thin films in a LbL fashion using copper(I)catalyzed azide-alkyne [3+2] cycloaddition (CuAAC) reactions (commonly referred to as "click" chemistry) as both a means to link the layers together and attach them to the substrate surface. $^{23,24}$ "Click" chemistry or CuAAC, discovered by Sharpless ${ }^{25}$ and Meldal, ${ }^{26}$ has been popularized as a rapid, facile, and robust cycloaddition reaction utilizing inexpensive $\mathrm{Cu}(\mathrm{I})$ catalyst. CuAAC selectively generates a 1,4-triazole linkage between terminal acetylenes and azides. This simple reaction is tolerant of a variety of conditions and other functional groups, and has been used extensively as a surface modification technique on multiple surfaces. ${ }^{27-34}$ Although a few other groups have used this reaction to fabricate triazole-linked polymer-based multilayers, ${ }^{35-38}$ to the best of our knowledge, we were the first to report the use of CuAAC to build molecular multilayer films. ${ }^{23}$ Since then, others

\section{Received: August 19, 2011}

Accepted: October 26, 2011 


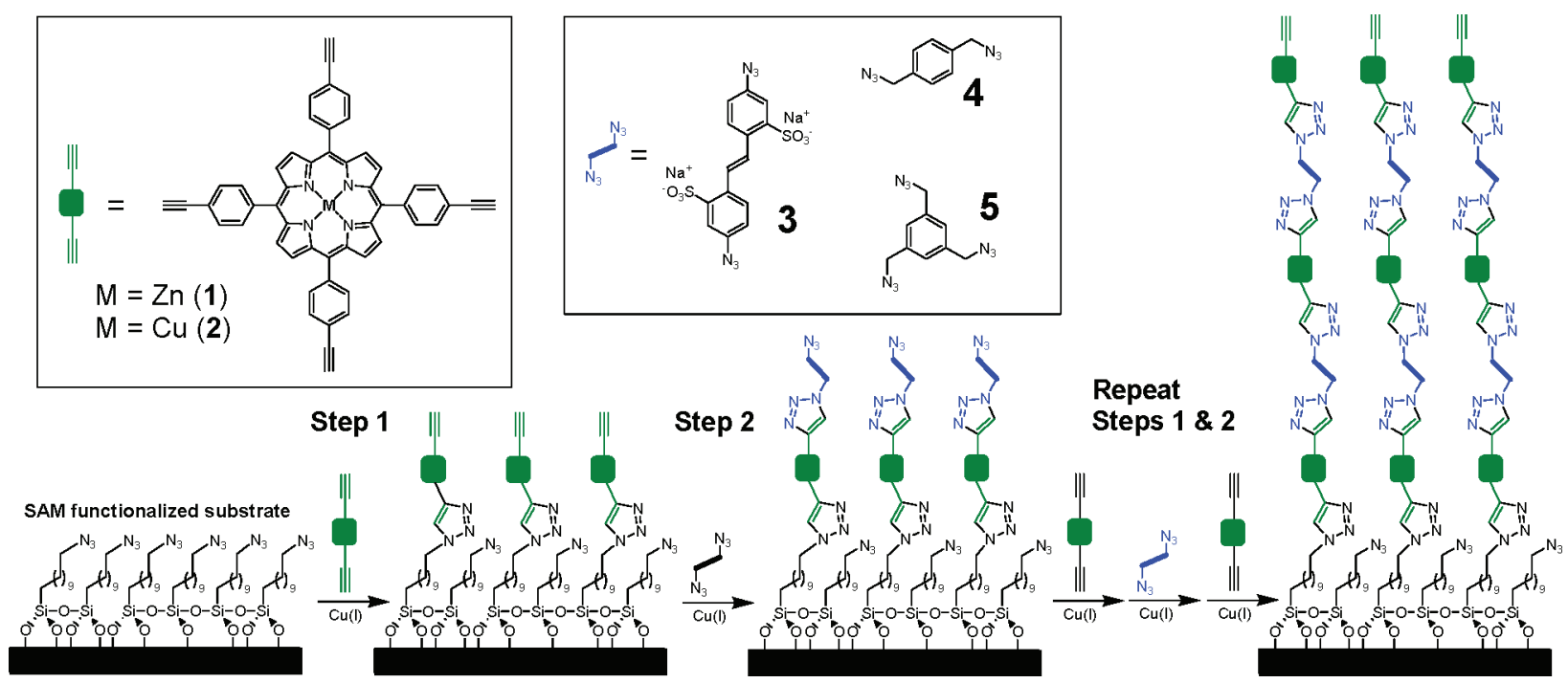

Figure 1. Outline of molecular LbL multilayer growth using CuAAC reactivity.

have used CuAAC for similar LbL generation of triazole-based molecular wires on $\mathrm{Au}(111)$ electrodes. ${ }^{39}$

Our approach to using CuAAC for molecular LbL fabrication is outlined in Figure 1. The methodology is similar to other $\mathrm{LbL}$ schemes, utilizing two sequential self-limiting reactions that add a monolayer of the primary molecular building block and a linker group to the surface at each step. The CuAAC-based multilayer fabrication process begins with the functionalization of an oxide surface with an azido-terminated alkyl siloxane to form a selfassembled monolayer (SAM). Next a multiethynyl molecule, such as $\mathbf{1}$ or $\mathbf{2}$, is reacted with the azide-terminated SAM in the presence of $\mathrm{Cu}(\mathrm{I})$ catalyst to generate a densely packed monolayer of 1,4-triazole linked porphyrins and a surface that is now terminated with ethynyl moieties (step 1). Finally, the reaction of a multiazide molecule, such as 3, 4, or 5 (herein referred to as linkers), with the ethynyl functionalized surface completes a molecular bilayer and regenerates a surface rich with azide groups for continued multilayer growth (step 2). These two self-limiting reactions are repeated sequentially to generate a multilayer film quickly and easily. We are specifically interested in porphyrin containing films due to their high molar absorptivities and tunable electrochemical and photophysical properties. ${ }^{40}$ Porphyrin-based LbL films could be useful in application such as molecular electronics, ${ }^{41,42}$ artificial photosynthesis, ${ }^{43}$ semiconductor sensitization, ${ }^{4-47}$ and sensors. ${ }^{48}$ Our work to better understand the molecular orientation, bonding motif, electrochemical and photophysical properties of these films will aid in tuning and optimizing film properties to the desired application.

Multilayer films fabricated by this method show linear and reproducible growth trends in absorbance and thickness through tens of layers and have the ability to incorporate various molecular building blocks including porphyrins, perylene diimides, and several azido linkers. ${ }^{23,24}$ Our initial results indicate that there is a strong correlation between the film thickness and the length of azido linker used. Films grown with 1 and 3 result in thicker films compared to films grown with $\mathbf{1}$ and $\mathbf{5}$ (2.41 vs $1.87 \mathrm{~nm} /$ bilayer). We estimated a range of growth angles for the films based on X-ray reflectivity and ellispometrically derived thicknesses and intramolecular distances from DFT calculated structures. These experiments could provide only a range of molecular growth angles due to several possible binding modes with the tetra-ethynyl functionalized porphyrin (1). Additionally, little information was available regarding the yield of azide to triazole conversion in the film. To predict various structural, electrochemical, and photophysical properties of the films, we require a better understanding of the primary binding modes present in the films resulting from $\mathrm{CuAAC}$ based $\mathrm{LbL}$ growth. Toward this end we employed grazing-angle attenuated total reflectance (GATR) IR spectroscopy and polarized UV-vis spectroscopy to determine the average growth angle of the porphyrin building blocks in the multilayer structures. These results coupled with previous estimates of the growth angle based on optimized structures of the porphyrin-linker subunits provide greater insight into the molecular structure within the multilayer film. Additionally, we compare the electrochemical responses of the films to both the UV-vis absorbance and GATR IR trends observed during multilayer growth to gain insight into the structure and density of molecular building blocks within the film.

\section{RESULTS AND DISCUSSION}

Infrared Analysis. Polarized grazing-angle attenuated total reflectance (GATR) FT-IR was employed at each step throughout multilayer growth to confirm surface attachment of the molecular components through CuAAC as outlined in Figure 1. Figure 2 shows a selection of GATR IR spectra taken throughout the fabrication of a multilayer thin film comprised of 1 and 3 . An intense azide stretch at $2100 \mathrm{~cm}^{-1}$ as well as the typical $\mathrm{CH}$ stretches at $2934 \mathrm{~cm}^{-1}$ and $2859 \mathrm{~cm}^{-1}$ (not shown) are observed for the base of the multilayer structure, an azide terminated $\mathrm{C}_{11}$ alkyl siloxane SAM anchored to an indium tin oxide (ITO) surface (black line, Figure 2). Upon reaction of porphyrin 1 with the azido-SAM, the intensity of the $2100 \mathrm{~cm}^{-1}$ stretch decreases drastically, consistent with the conversion to triazoles via a $\mathrm{CuAAC}$ reaction with 1 (red line, Figure 1). It is difficult to assign any of the peaks to the triazole $\mathrm{C}-\mathrm{H}$ stretching mode due to the convolution of aromatic $\mathrm{C}-\mathrm{H}$ peaks from the porphyrin and linkers centered around $1600 \mathrm{~cm}^{-1}$. 39 Additionally, the presence of intense bands at 797 and $998 \mathrm{~cm}^{-1}$, corresponding to $\beta$-hydrogen out-of-plane deformation and the in-plane 


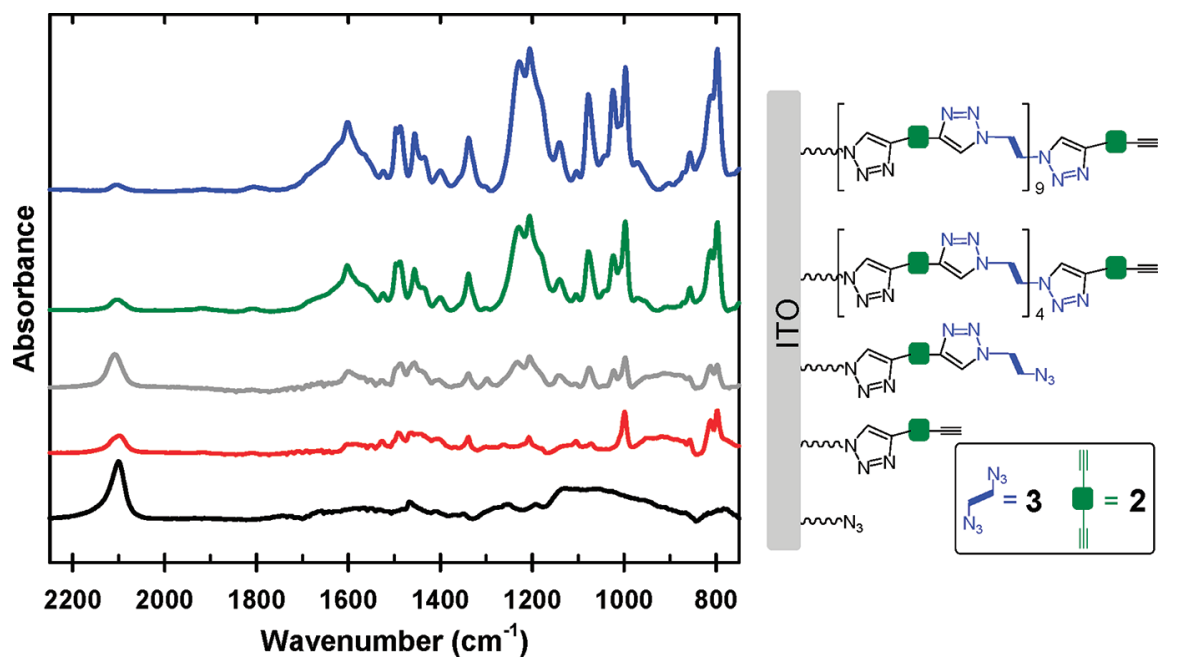

Figure 2. GATR FT-IR spectra resulting from different stages during multilayer growth of films containing 1 and 3 on ITO. Spectra are offset for clarity.

breathing mode of porphyrins respectively, ${ }^{49,50}$ indicate that $\mathbf{1}$ has reacted with the azide-terminated SAM. Upon reaction of multi-azide containing linker molecule, 3 , the intensity of the peak at $2100 \mathrm{~cm}^{-1}$ increases suggesting that at least one of the azides from the linker has reacted with the ethynyl groups of $\mathbf{1}$ leaving the others to continue multilayer growth in the next step. Similar trends are also observed for multilayers fabricated using porphyrin 2 and linkers 4 and 5. New peaks are observed upon addition of the azide-containing linker including one at $1023 \mathrm{~cm}^{-1}$ and a broad set of peaks around $1200 \mathrm{~cm}^{-1}$ which are most likely due to the sulfonate groups on $3 .{ }^{51}$ With the exception of the azide stretch, the peaks resulting from the linker and porphyrin increase systematically with the number of layers added in the film implying that the concentration of molecular units increases with the number of layers that are grown.

The intensity of the azide stretch at $2100 \mathrm{~cm}^{-1}$ shows an oscillating trend as a function of the particular step in the multilayer fabrication and the number of layers added to the film. The oscillating pattern seen in Figure 3 is consistent with the multilayer fabrication method outlined in Figure 1 and strongly depends on the particular linker used in multilayer growth. The intensity of the $2100 \mathrm{~cm}^{-1}$ is large for the 11-azidoundecyltrimethoxysilane SAM coated ITO electrode due to the high surface density of terminal azides. Following the CuAAC reaction to form a monolayer of porphyrin, the azide peak decreases drastically, but does not disappear completely. This is due to the fact that the density of azides present on the SAM functionalized surface is greater than the number of porphyrin molecules that can pack on the surface (vide infra). With the addition of a linker layer the azide stretch increases again, but does not reach the level of the original SAM implying that the density of the azides at the linker layer is less than what was originally present for the SAM. The penetration depth of IR light in the GATR technique is significantly greater $(0.5-1 \mu \mathrm{m})$ than the thickness of the films $(2-25 \mathrm{~nm})$, and thus the spectrum is taking into account all of the azides present in the multilayer film. ${ }^{52}$ Thus, the intensity of the $2100 \mathrm{~cm}^{-1}$ peak includes unreacted azides remaining from the SAM as well as the azides present from the addition of the top-most linker layer.

Figure 3 shows the value of the integrated azide stretch at $2100 \mathrm{~cm}^{-1}$ versus the number of layers added for the three different linkers used in this study. ${ }^{53}$ The two bisazide linkers 3 and 4

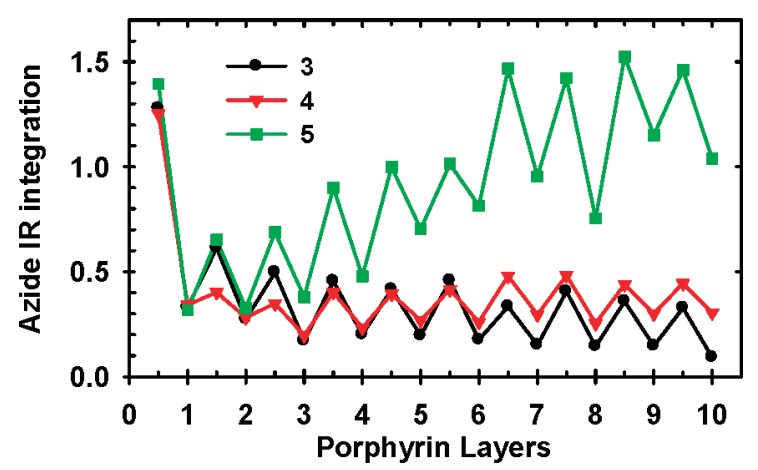

Figure 3. Azide content in films of 1 with linkers 3,4 , and 5 as determined by GATR IR. Whole number bilayers indicate the addition of porphyrin $\mathbf{1}$, whereas the linker layers are represented as $1 / 2$ layers.

(black circles and red triangles, respectively) show an oscillating trend in azide integration that increases with addition of a linker layer and decreases with the porphyrin layer but show a net slope of nearly zero. Mesitylene linker 5 (green squares) has three azide moieties and shows a similar oscillating pattern in the azide integration, however, the overall slope of the plot is clearly positive beyond the first layer. We attribute this to unreacted azides left over from linker 3 building up over the course of multilayer growth. Multilayers that incorporate 3 or 4 most likely react both of the azide groups present in high yield - one with the ethynyl layer below and one above, resulting in no net increase of azide content in the film. Linker $\mathbf{5}$ has three azide units, thus there is one remaining after the other two have reacted with porphyrin layers below and above the layer of $\mathbf{5}$. The usability and accessibility of the remaining azide is discussed later.

UV-Vis and Infrared Spectroscopic Trends. Figure 4 shows a representative set of $\mathrm{UV}$ - vis spectra taken during the assembly of a multilayer of 1 with linker 3 . The absorbance of the Soret and Q-bands increase with the numbers of porphyrin layers added to the film. As discussed previously, ${ }^{2 \frac{3}{3}}$ the Soret from $\mathbf{1}$ in a multilayer environment is red shifted and broadened compared to the solution spectra, indicating closely packed J-aggregated porphyrins. ${ }^{54}$ It is interesting to note that the absorbance trends observed for multilayers grown using $\mathbf{1}$ with linkers $\mathbf{3}$ or $\mathbf{5}$ show 

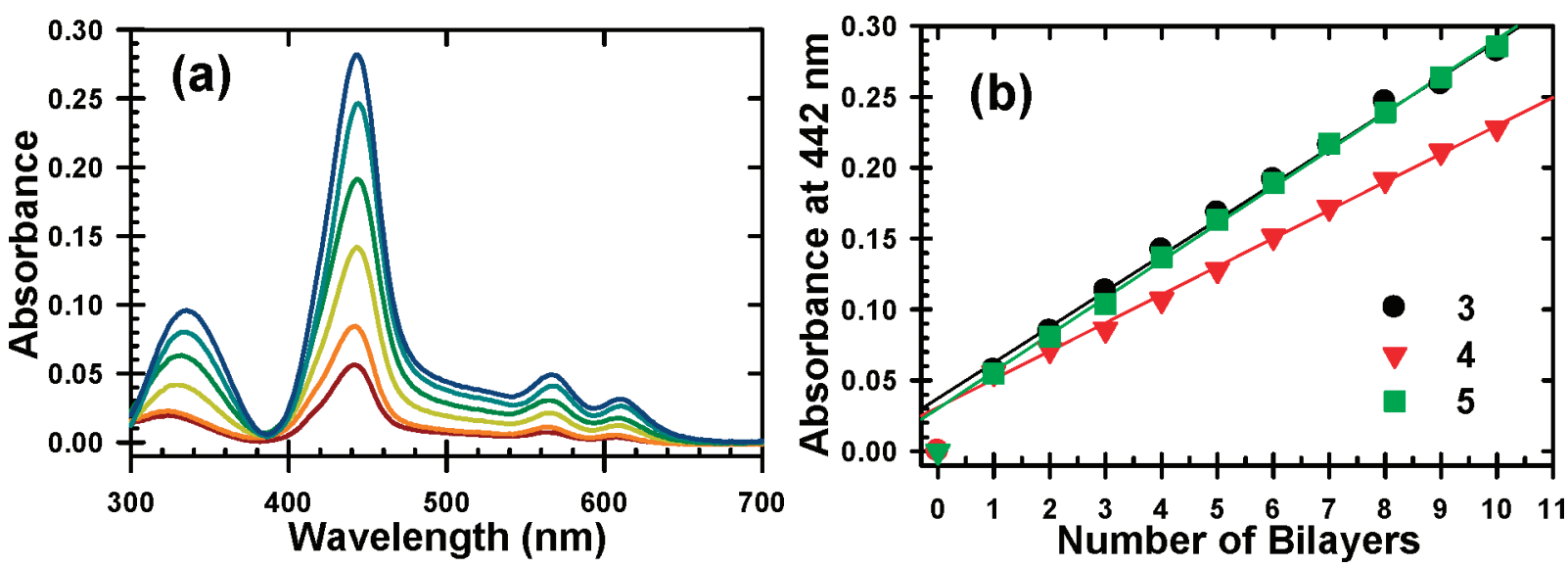

Figure 4. (A) Absorbance of a multilayer of 1 and 3 on ITO at 1, 2, 4, 8, and 10 bilayers. (B) Absorbance at Soret max (442 nm) for multilayers grown with porphyrin 1 and linkers 3,4 , and 5 . Trend lines exclude the first data point ( 0 bilayers).

Table 1. Comparison of Linkers Used in This Study

\begin{tabular}{cccc} 
& no. of & $\begin{array}{c}\text { azide IR trend } \\
\text { after first layer } \\
\text { (Figure 2) }\end{array}$ & $\begin{array}{c}\text { absorbance growth } \\
\text { at 442 } \mathrm{nm} \text { after first layer } \\
\text { (mAU/bilayer) }\end{array}$ \\
\hline stilbener 3 & 2 & oscillating, overall flat & 25 \\
xylene 4 & 2 & oscillating, overall flat & 20 \\
mesitylene 5 & 3 & oscillating, overall increasing & 26 \\
\hline
\end{tabular}

very similar increases in absorbance after the first layer $(25$ and 26 $\mathrm{mAU} /$ bilayer respectivly, $\mathrm{mAU}=$ milliabsorbance units) while the film containing 1 and 4 grew at a lower rate $(20 \mathrm{mAU} /$ bilayer, Figure 4). The relatively large increase in absorbance for the first bilayer (>50 mAU) compared to the other layers is consistent with changes in azide peak intensity observed by IR. A large decrease in azide IR intensity corresponds to a large increase in absorbance for the porphyrin monolayer (CuAAC reaction with 1 will result in loss of azide and generation of triazole linked porphyrin). The smaller increases in absorbance for subsequent multilayer steps (20 mAU/bilayer for films of 1 and 4, $25 \mathrm{mAU} /$ bilayer for films of 1 and 3, and $26 \mathrm{mAu} /$ bilayer for films of $\mathbf{1}$ and 5) correlate to a smaller change in the oscillating azide IR peak intensity (Figure 1). This is likely due to the high concentration of azides present for the 11-azidoundecyltrimethoxysilane SAM modified substrate, allowing for the formation of a relatively dense porphyrin monolayer when $\mathbf{1}$ is reacted with the surface. Upon reaction of the ethynyl-terminated surface with the linker molecules, the azide content is not as high as the initial SAMmodified surface. This lower azide density of the linker layers result in subsequent porphyrin layers that are not as dense as the first, and thus the lower rate of increase in absorbance. From the addition of the second porphyrin layer on, the trend in absorbance is clearly linear suggesting that the packing of the molecular layers remains constant beyond the first monolayer of porphyrin. Following is a detailed comparison of UV-vis and IR trends for films grown with various linkers summarized in Table 1.

A comparison of the UV-vis absorbance to the azide IR trend (Figure 5) shows the correlation between absorbance and azide content in the film. Upon reaction of $\mathbf{1}$ with the azide terminated SAM, the absorbance at the Soret maximum increases (blue triangles, 0.5 bilayers) while the $2100 \mathrm{~cm}^{-1}$ peak for the asymmetric azide

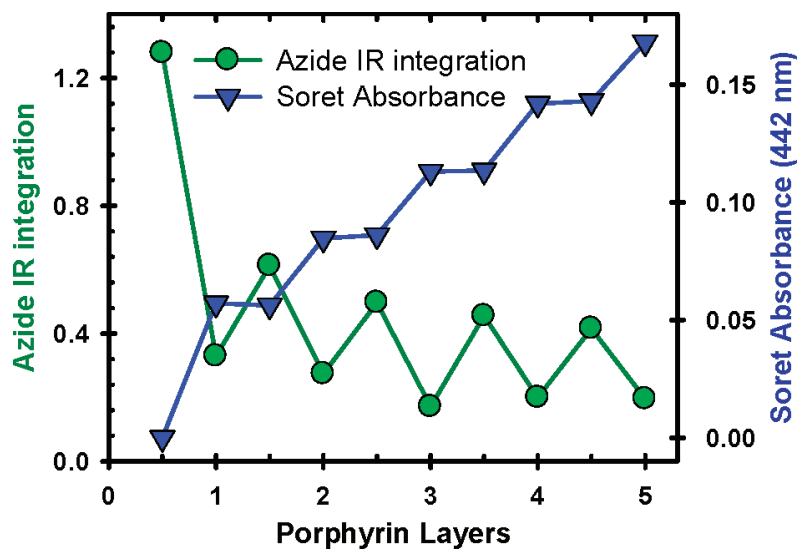

Figure 5. Comparison of the absorbance (blue triangles) and azide IR integration (green circles) of a multilayer of $\mathbf{1}$ and $\mathbf{3}$ on ITO.

stretch decreases (green circles, 0.5 bilayers) as expected for triazole formation via CuAAC. When linker 3 is reacted with the ethynyl-terminated porphyrin layer, there is no increase in absorbance, however, there is a significant jump in the $2100 \mathrm{~cm}^{-1}$ IR peak ( 1 bilayer). This increase in the $2100 \mathrm{~cm}^{-1}$ IR peak is due to the azides from linker 3 that are now present at the peripheral of the film. The peak at $2100 \mathrm{~cm}^{-1}$ does not return to its original intensity because the density of azides present on the SAM (layer 0) is much greater than the density of azides present after reaction with linker 3. Addition of a second layer of porphyrin results in an increase in absorbance and decrease in azide content. The absorbance increase (much like the azide trend) does not increase as much as it did during the initial porphyrin reaction with the azido-SAM. This is again due to the fact that there are not as many azides on the surface available for reaction with the porphyrin which results in a smaller increase in absorbance. Subsequent layers show a linear increase in absorbance upon addition of every porphyrin layer, and the azide content in the film continues to show an oscillating trend.

Comparison of Linker 3 and 4. A comparison of multilayers of 1 with either bisazide linkers 3 and 4 reveals an inherent difference in their growth patterns. Multilayers grown with 4 results in films that have a lower growth rate than those assembled with linker 3 (20 vs $25 \mathrm{mAU} /$ bilayer). Multilayers grown using 


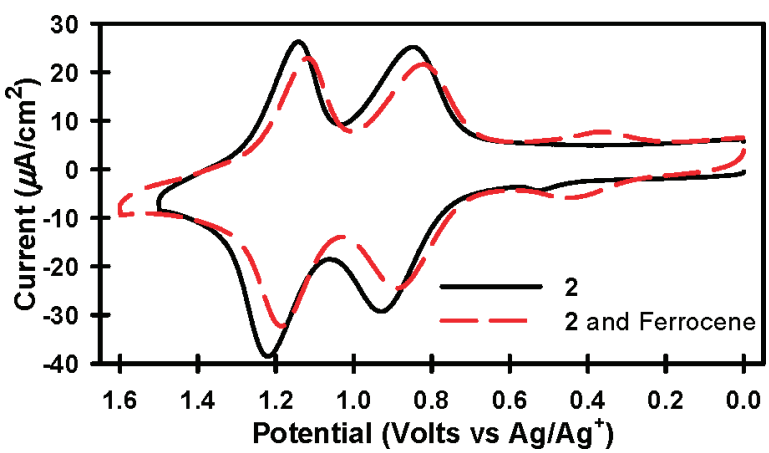

Figure 6. Cyclic voltammogram of a monolayer of porphyrin $\mathbf{2}$ on ITO before (black, solid line) and after (red, dashed line) CuAAC reaction with ethynylferrocene. CVs were taken at scan rate of $200 \mathrm{mV} / \mathrm{s}$ with $0.1 \mathrm{M}$ TBAP in dichloromethane as the electrolyte.

stilbene linker 3 have a larger oscillating trend in the azide IR stretch as well. This implies that the films have a higher porphyrin density and the reaction yield at the linker layer is greater for 3 than for $\mathbf{4}$. Given the structure of $\mathbf{4}$ which contains methylene units between the aromatic core and reactive azide groups, it is possible that both azides of linker 4 can react with the lower porphyrin layer (a capping event), leaving fewer azides available for continuation of the multilayer. Linker 3 is a more rigid structure with the reactive azide groups directly connected to the phenyl rings (i.e., less rotational flexibility). This may limit linker 3 to react only one azide with the lower porphyrin layer, thus limiting capping events and ensuring continued multilayer growth.

Comparison of Linker 3 and 5. Multilayers grown with linkers 3 and $\mathbf{5}$ display very similar increases in absorbance $(25$ and $26 \mathrm{mAU} /$ bilayer respectively) suggesting that the films have comparable porphyrin surface densities. Given the structural differences between linkers $\mathbf{3}$ and $\mathbf{5}$, it is safe to assume that the porphyrin size is dictating the rate of film growth. The obvious difference between the films grown with these two linkers is the azide content of the film. Films containing linker 3 show an oscillating trend in the azide content (as determined by IR) but the overall trend remains flat. Conversely, films containing linker $\mathbf{5}$ show a steady increase in the overall azide content as well as an oscillation in azide IR intensity between the porphyrin and linker layers.

Comparison of Linker $\mathbf{4}$ and $\mathbf{5}$. Linkers $\mathbf{4}$ and $\mathbf{5}$ are the most similar of the three linkers structurally, but they result in very different films. The film grown using linker 4 results in a multilayer with $80 \%$ of the porphyrin content (by absorbance) of the film grown using linker 5. The increase in porphyrin content may be attributed to the extra azide that is present on linker $\mathbf{5}$ allowing for dendritic type growth as well as the presence of one remaining azide if two happen to react with the lower porphyrin layer (a "capping" event). It is clear from IR that the third azide present is not always utilized as indicated by the buildup of azide in the film containing linker 4 . Films grown with 5 do not show a buildup of azide content which is expected for any bisazide linker because both azides are utilized in the LbL process. The difference in intramolecular distance between azides is also slightly different for linkers $\mathbf{4}$ and $\mathbf{5}$ which could contribute to the difference in growth trends. The para orientation of functional groups in 4 results in azides that are further apart than those in a meta orientation of $\mathbf{5}$. This may result in a larger portion of linker 4 participating in a capping events, leading to lower growth rates. The high number of functional groups found

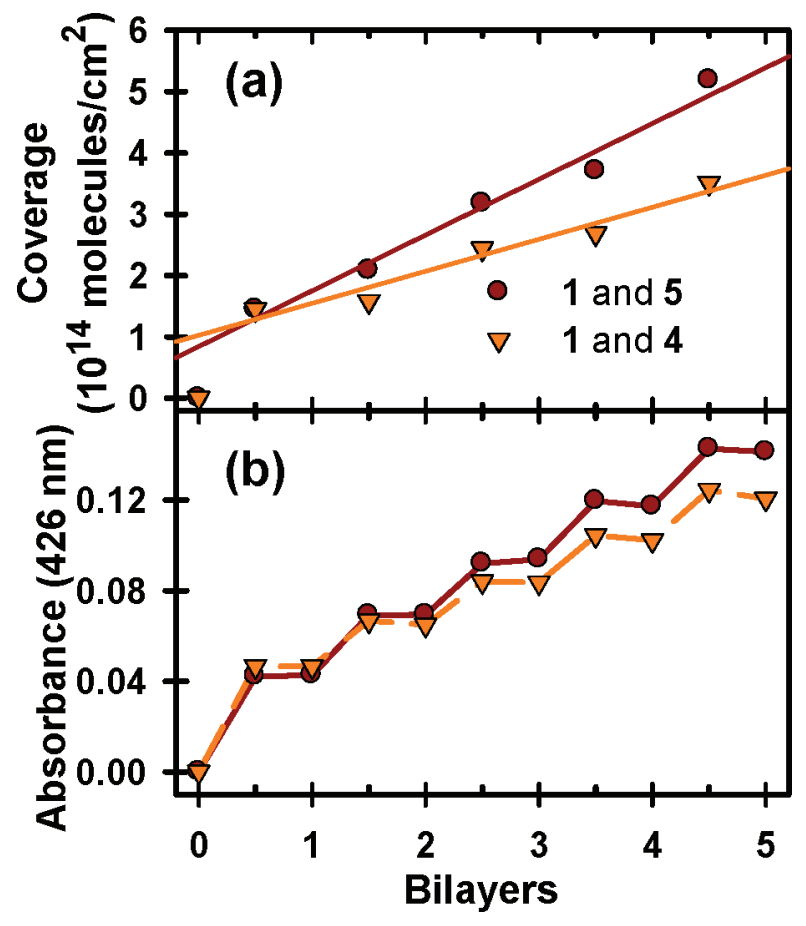

Figure 7. (a) Coverage as determined by integration of the charge passed in cyclic voltammograms of $1-5$ bilayers of porphyrin 2 with linker $\mathbf{5}$ or $\mathbf{4}$ and ethynyl ferrocene. Trend lines exclude the first data point for zero porphyrin layers. (b) Absorbance trends of the same multilayers at the Soret maximum (426 nm).

in porphyrin $\mathbf{1}$ and linker $\mathbf{5}$ could allow multilayer growth to overcome capping events through dendritic growth, which would help maintain a high porphyrin packing density at each layer.

Electrochemical Determination of Porphyrin Coverage. Electrochemical investigation of the multilayer films provides a straightforward method to determine surface coverage of porphyrins through integration of the charge passed for the two one-electron oxidation waves in the cyclic voltammograms $(\mathrm{CV})$ via eq $1 .^{55}$

$$
\Gamma=\frac{Q}{n F A}
$$

In eq $1, \Gamma$ is the surface coverage of the redox active groups per unit area, $Q$ is the integrated charge of Faradic response resulting from porphyrin oxidation on the surface, $n$ is the number of electrons involved, $F$ is Faraday's constant, and $A$ is the active area of the electrode. ${ }^{55}$ The area of the ITO electrode was scaled using a factor of 1.08 to account for the roughness of the ITO surface. ${ }^{56}$

Both $\mathrm{Cu}^{\mathrm{II}}$ and $\mathrm{Zn}^{\mathrm{II}}$ meso-tetraphenylporphyrins typically display two single electron oxidation waves at moderate potentials. ${ }^{57}$ Representative CVs of multilayer films comprised of 2 with linkers 4 and 5 are shown in Figure 6 and Figure S1 in the Supporting Information, respectively. ( 2 was selected over $\mathbf{1}$ for the electrochemical analysis due to the slight anodic shift of the oxidation potentials to allow for a clear potential window in which to estimate ethynylferrocene incorporation into the films (vide infra).) Figure 7a shows the calculated coverage as a function of the number of bilayers for multilayers grown with 2 and linkers 4 and 5. Similar to what was seen in absorbance trends for films grown with porphyrin 1 (Figure 4), multilayer assembled with linker $\mathbf{5}$ grows at a higher rate than those with linker 4 after the initial increase for the first porphyrin layer. We also observe a 
greater increase in coverage for the first monolayer of porphyrin deposited on the SAM than in subsequent bilayer reactions (Figure 7a). This is similar to the trend observed in UV-vis absorbance spectra (Figure $7 \mathrm{~b}$ ).

Monolayers of $\mathbf{1}$ attached to the 11-azidoundecylsiloxane coated ITO electrode gave a surface coverage of $1.44 \times 10^{14}$ molecules $/ \mathrm{cm}^{2}$, which corresponds to a molecular footprint area of $69 \AA^{2}$. This footprint is close to the estimated size of the clicked porphyrin of $75 \AA^{2}$ assuming a rectangular box encompassing the van der Waals distance at the extremes of the DFT minimized molecular structure. ${ }^{24}$ This footprint is similar to other reports for densely packed monolayers of tetraphenylporphyrins on a $\mathrm{Si}(100)$ surface having a molecular footprint of $75 \AA^{2} \cdot{ }^{16,58}$ To estimate the number of azides available for reaction at the 11-azidoundecylsiloxane coated ITO electrode surface, we attached ethynyl ferrocene via $\mathrm{CuAAC}$ and achieved a coverage of $1.68 \times 10^{14}$ molecules $/ \mathrm{cm}^{2}$. This resulted in a footprint area of $59.5 \AA^{2}$, which is larger than expected for a dense packed monolayer based on the size ferrocene $\left(34 \AA^{2}\right) .{ }^{59}$ However, this coverage is larger than the coverage attained with porphyrin $\mathbf{1}$ or 2. GATR IR analysis of the ethynylferrocene clicked surface revealed that the IR peak at $2100 \mathrm{~cm}^{-1}$ did not reduce to zero suggesting that the ferrocene is not able to access all of the azides despite its smaller size compared to porphyrins $\mathbf{1}$ and 2. This indicates that under the $\mathrm{CuAAC}$ reaction conditions employed herein, the ethynyl terminated molecules are not able to access all of the azides on the surface, explaining the remaining azide observed in the IR measurements (see Layer 1, Figures 3 and 5). It is also possible that the CuAAC catalyst size, not just ethynylcontaining molecule, is preventing access to all of the azides on the surface. Proposed CuAAC mechanistic cycles suggest the copper(I) catalyst forms a Cu-acetylide complex as a precursor to the cycloaddition reaction. ${ }^{25,60}$ The bulky $\mathrm{Cu}(\mathrm{I})$ stabilizing ligand (tris-(benzyltriazolylmethyl)amine, TBTA) used in our reaction conditions could be imposing additional steric constraints, thus restricting maximum surface coverage of both ethynylferrocene and porphyrins,

Porphyrin Orientation. GATR IR spectroscopy can be used to determine the average molecular orientation of the porphyrin plane within the monolayers and multilayer thin films by comparing the ratio of vibrational intensities for the in-plane breathing mode of the porphyrin at $997 \mathrm{~cm}^{-1}$ versus the $\beta$-hydrogen out-of-plane deformation at $797 \mathrm{~cm}^{-1} \cdot 16,49,50,61$ These two orthogonal features allow us to calculate the average molecular orientation of the porphyrin plane $(\theta)$ by comparing the intensity of these two IR peaks in the film environment to that of an isotropic sample ( $\mathrm{KBr}$ pellet) using eq $2^{16,62,63}$

$$
\tan ^{2} \theta=\frac{I_{997}^{\text {bulk }}}{I_{797}^{\text {bulk }}} \frac{I_{997}^{\text {film }}}{I_{997}^{\text {film }}}
$$

In eq 2 , the orientation of porphyrin plane with respect to substrate surface is $90-\theta$ and $I$ represents the relative intensities of the 997 and $797 \mathrm{~cm}^{-1}$ peaks in the bulk and film environments. Figure 8 shows the difference between the transmission IR spectrum of a sample of $\mathbf{1}$ in $\mathrm{KBr}$ along with a GATR FT-IR spectrum of a multilayer of $\mathbf{1}$ and $\mathbf{5}$ on ITO. One noticeable difference is the increase in the ratio between the 997 and $797 \mathrm{~cm}^{-1}$ peaks from the isotropic IR spectrum (Figure 8 solid black line) to the surface IR spectrum (Figure 8 dashed red line). This difference is due to the preferred orientation that the porphyrin maintains in the film environment. Using eq 2 , the

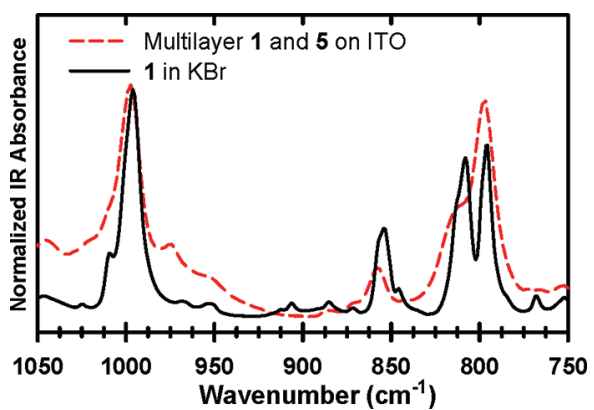

Figure 8. Comparison of the IR spectrum of porphyrin 1 in $\mathrm{KBr}$ (random orientation) and in multilayer film environment with $\mathbf{5}$ as the linker.

calculated average angle of the porphyrin plane with respect to the surface for 5,10 , and 15 bilayer films of 1 with either linker 3 or $\mathbf{5}$ is $44^{\circ}$. A monolayer of $\mathbf{1}$ on ITO results in a film with a calculated porphyrin angle of $45^{\circ}$ by GATR IR. While the angle difference between the initial layer and subsequent bilayers is small, and probably within the error in measurement technique, the higher angle is consistent with previous $\mathrm{UV}$-vis absorbance and electrochemical observations that the initial monolayer has a higher porphyrin coverage than the following bilayers. The higher coverage for the monolayer would require a more vertical orientation of the porphyrins within the film.

Polarized absorbance spectroscopy was used as a secondary method to confirm the orientation of the porphyrins in the thin film environment. Polarized UV - vis spectroscopy has been used previously as a simple and effective way of determining the average molecular orientation with respect to the surface of porphyrin and phthalocyanine in different film environments. ${ }^{64-66}$ Samples oriented $45^{\circ}$ versus the incident light beam are subjected to horizontally and vertically polarized light. Figure 9 shows sample spectra of a multilayer of $\mathbf{1}$ and $\mathbf{5}$ on ITO with the polarization angle varied from 0 (vertically polarized) to 90 (horizontally polarized). It is obvious from the absorption spectra that the films have some orientation dependence. The average molecular orientation can be found using eq 3 where the dichroic ratio, $D(\lambda)$, is equal to the ratio of the absorbance when the light is horizontally polarized to the absorbance when the light is vertically polarized $\left(A_{h} / A_{v}\right)$. $^{64-66}$ The angle of the porphyrin plane (and the electronic dipole moment vector for the Soret) with respect to the substrate surface $(\gamma)$ can be calculated using eq 3 , and is found to be $47^{\circ}$ for multilayers containing 1 and 3 (see Figure S2 in the Supporting Information for polarized absorption spectra) and $45^{\circ}$ for multilayers containing $\mathbf{1}$ and $\mathbf{5}$.

$$
D(\lambda)=\frac{A_{h}}{A_{v}}=\frac{2 \sin ^{2} \alpha \sin ^{2} \gamma}{2-\sin ^{2} \gamma}+\cos ^{2} \alpha
$$

When the substrate is oriented normal to the incoming light, there is no polarization dependence $(D(\lambda)=1)$, which shows that there is no orientation preference in the plane of the substrate (random orientation around an axis normal to the surface). This is expected to be the case because the solution deposition method used in this study should not induce a preferred direction in the plane of the substrate.

A range of $41-70^{\circ}$ was estimated for the growth angle of the porphyrin within the films in our previous work through comparison of experimentally determined film thickness and molecular modeling. ${ }^{24}$ The calculated range of $45-47^{\circ}$ from polarized 

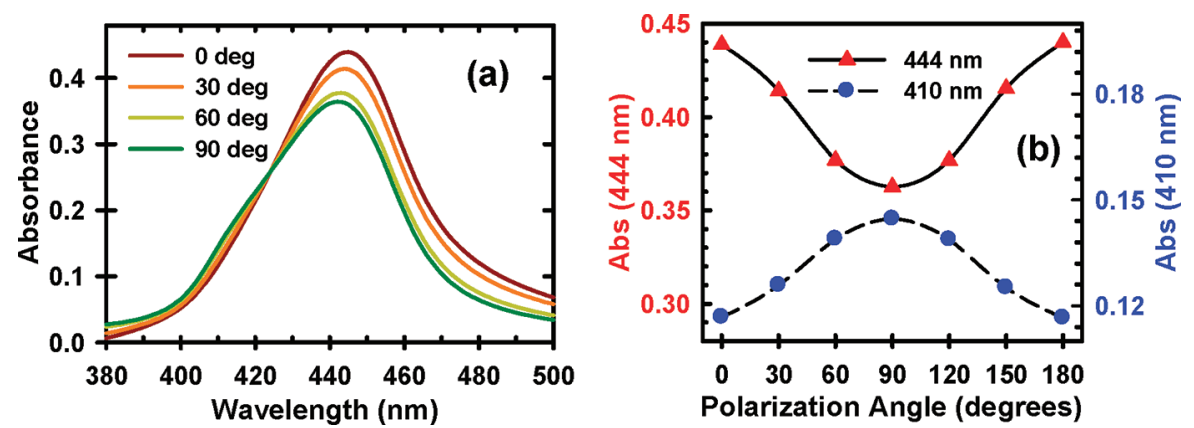

Figure 9. (a) Polarized absorbance spectra for the Soret band of the porphyrin in a film containing 15 bilayers of $\mathbf{1}$ and 5 on ITO. (b) Absorbance dependence on polarization angle at 444 and $410 \mathrm{~nm}$.

UV-vis spectra in this work match GATR FT-IR results and are near the lower end of the estimates for the growth angle of our previous estimate (growth angles are summarized in Table 2). ${ }^{24}$ Two bonding modes can be envisioned for tetra-ethynyl porphyrin $\mathbf{1}$ and $\mathbf{2}$ that would result in linear LbL growth (Figure 10): A trans bonding mode where the ethynyl groups that are across the porphyrin from one another are the only groups reacting, or a cis bonding mode where two ethynyl groups that are adjacent to one another are both reacting with the linker or SAM below, allowing for two linkers to react with the remaining ethynyl groups of porphyrin 1 . The trans bonding mode will result in a longer dimer length (a dimer is defined as one porphyrin-linker repeat unit) which would give rise to a lower porphyrin plane angle with respect to the surface. The cis bonding mode would result in a shorter dimer length and a more vertical average porphyrin angle. The similarity between the average porphyrin angles when either $\mathbf{3}$ or $\mathbf{5}$ is used suggests that the linkers may not dictate the angle at which the porphyrin multilayers grow. Rather, the maximum packing density of the porphyrin is most likely the dominating factor in controlling the growth angle. Our findings herein suggest that the films grown using porphyrin $\mathbf{1}$ with linker $\mathbf{3}$ or $\mathbf{5}$ contain predominantly a trans bonding motif.

Studies of porphyrin monolayers attached to a $\mathrm{Si}(100)$ surface through a tripodal allyl tether result in average porphyrin plane orientations between 49 and $54^{\circ}$ with respect to the surface as determined by $\mathrm{IR}^{21}$ An average porphyrin angle of $55-60^{\circ}$ has been determined for multilayers of tetra-pyridyl porphyrins constructed via Pd-pyridine coordination using polarized UVVis spectroscopy. ${ }^{66}$ The angles we have determined for mono and multilayers of porphyrin $\mathbf{1}$ on ITO are lower than those determined by others, however, our LbL scheme is quite different than those used for other $\mathrm{LbL}$ processes. The CuAAC based $\mathrm{LbL}$ assembly technique along with the templating effect of the azidoSAM are likely contributors to the discrepancy between our experimentally determined average porphyrin angles and those determined by others with similar systems.

Fabrication of Multicomponent Films. The GATR IR results obtained during the fabrication of multilayers grown using linker $\mathbf{5}$ indicate that there is a significant amount of unreacted azide remaining in the film (see Figure 3). To probe the accessibility of the remaining azides in the film following the addition of ethynylporphyrin layers, we exposed the films to a solution of ethynylferrocene and $\mathrm{Cu}(\mathrm{I})$ catalyst in hopes of reacting the redox probe with the remaining azide groups of 5 (see Figure S3 in the Supporting Information for schematic representation). When used in conjunction with $\mathbf{1}$, the ethynylferrocene redox potential is
Table 2. Calculated Values for Average Molecular Orientations of Porphyrin Plane with Respect to the Substrate Surface in Samples Containing 15 Bilayers of porphyrin 1 with linker 3 or 5

\begin{tabular}{lcc} 
& $\begin{array}{c}\text { average angle of } \mathbf{1} \text { in } \\
\text { multilayer with linker } \mathbf{3} \\
\text { method }\end{array}$ & $\begin{array}{c}\text { average angle of } \mathbf{1} \text { in } \\
\text { multilayer with linker } \mathbf{5} \\
(\mathrm{deg})\end{array}$ \\
$\begin{array}{l}\text { GATR FT-IR } \\
\text { GAlarized UV-vis }\end{array}$ & 44 & 44 \\
ref 24 (estimated range) & $46.7-70.3$ & 45 \\
\hline
\end{tabular}

too close to the first oxidation of porphyrin 1, making quantification of surface coverage by electrochemical methods somewhat difficult. For this reason, we used $\mathbf{2}$ in order to separate the redox potential of ferrocene and the porphyrin. The oxidation potentials of $\mathrm{Cu}$ (II) meso-tetraphenylporphyrins are $230 \mathrm{mV}$ more positive than $\mathrm{Zn}$ (II) analogs which allows for greater separation between the oxidation of ferrocene and $2 .{ }^{57}$ Figure 6 shows the CVs resulting from a monolayer of 2 before and after reaction with ethynylferrocene. Two one-electron oxidations are observed for a monolayer of 2 corresponding to a coverage of $1.5 \times 10^{14}$ molecules $/ \mathrm{cm}^{2}$. Following the additional CuAAC reaction of ethynylferrocene with the remaining azides, a smaller peak at $0.4 \mathrm{~V}$ appears due to the ferrocene moiety corresponding to a coverage of $1.38 \times 10^{13}$ molecules $/ \mathrm{cm}^{2}$ ( $8.4 \mathrm{~mol} \%$ ferrocene vs. 2 ). A plot of the ferrocene peak area versus scan rate results in a linear correlation, confirming that it is a surface bound species (see Figure S4 in the Supporting Information). ${ }^{55}$ GATR IR analysis of the resulting film shows the stretch at $2100 \mathrm{~cm}^{-1}$ also decreases in intensity demonstrating that the ethynylferrocene has accessed some of the remaining azide from the underlying SAM (see Figures S5 and S6 in the Supporting Information). However, the azide IR stretch does not disappear completely, which is expected due to the size of the porphyrin and ferrocene molecules compared to the spacing of the azides on the surface (see earlier discussion of molecular footprint and azide spacing on the surface). Once a full monolayer of porphyrin has reacted with the surface, ethynylferrocene can only access some of the remaining azide groups in the underlying SAM, resulting in a small decrease in the azide IR stretch at $2100 \mathrm{~cm}^{-1}$. The size of ethynylferrocene as well as the size of the $\mathrm{Cu}$ (I)TBTA catalyst may contribute to the inability to access all of the remaining azides on the SAM.

The additional CuAAC layer reaction with ethynylferrocene was continued throughout multilayer fabrication to evaluate the accessibility of remaining azides from linker $\mathbf{5}$. Following 


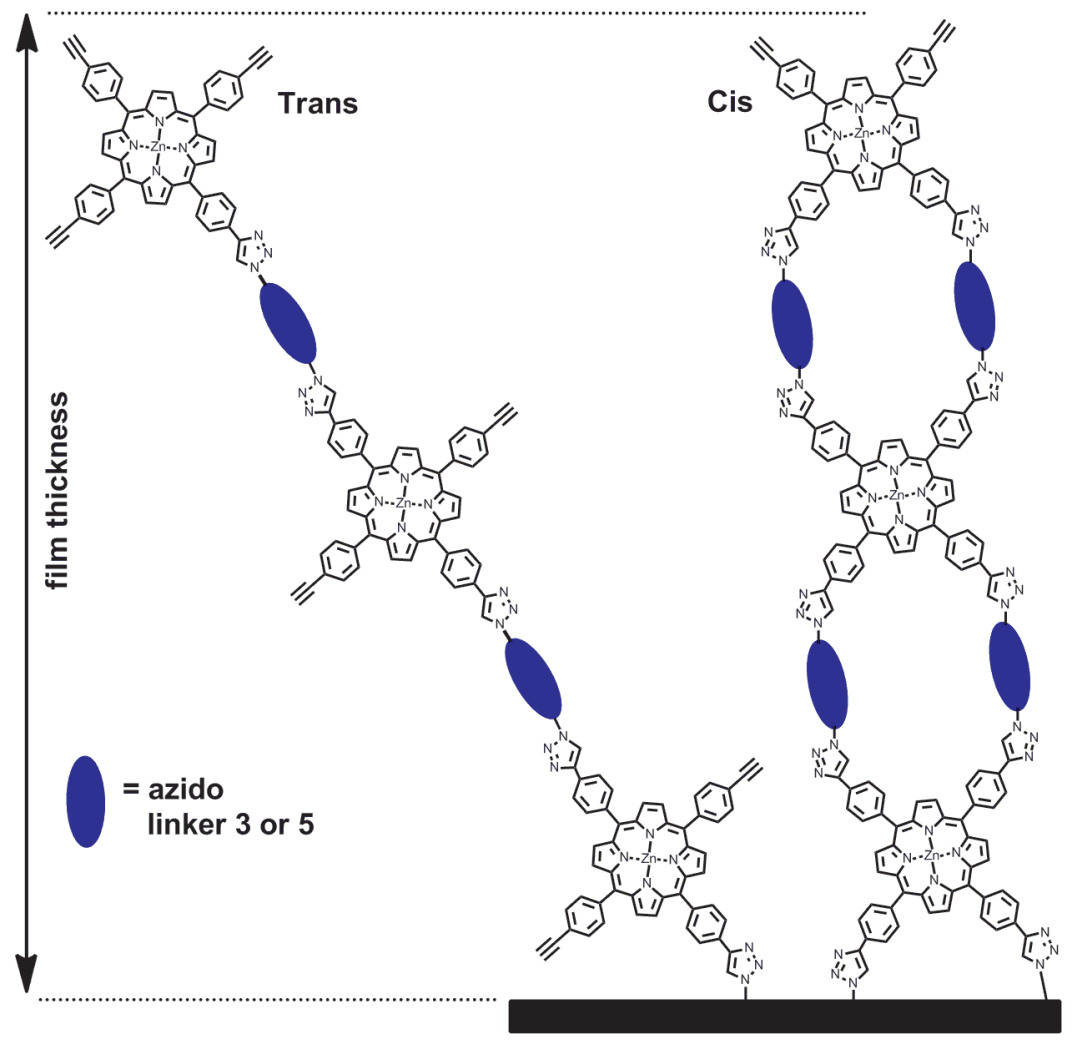

Figure 10. Depiction of trans and cis bonding modes in the multilayer films. With a known film thickness, a trans bonding mode will result in a lower average angle of the porphyrin plane with respect to the surface. Cis bonding mode will result in a more vertical orientation.

each CuAAC reaction with 2 to form the ethynyl-terminated porphyin layers, a second reaction was performed with ethynylferrocene and then finally the bilayer was completed with linker 5 (see Figure S3 in the Supporting Information). GATR IR results (see Figure S6 in the Supporting Information) confirmed that some of the azides from the underlying linker layer reacted with ethynylferrocene, but similar to what was observed above with the initial SAM layer, the decrease in the $2100 \mathrm{~cm}^{-1}$ peak was relatively small. This was consistent with electrochemical scans for the multicomponent films. Integrated CVs revealed average ferrocene incorporation of $8.45 \times 10^{12}$ molecules $/ \mathrm{cm}^{2}$, roughly $61 \%$ of the amount added after the first monolayer and only $9 \%$ of the amount of $\mathbf{2}$ added at each layer. This indicates that the porphyrin molecules are tightly packed throughout the film and do not allow significant penetration by ethynylferrocene and catalyst to the underlying azides from the linker layer. Thus, under the current conditions employed here, we were only able to generate a multicomponent film in which the second component, ethynylferrocene, was added at each layer in limited amounts. An ethynyl functionalized molecule with a different shape than ferrocene along with a smaller catalyst/ligand combination may improve the possibilities of generating multicomponent architectures with similar concentrations.

\section{CONCLUSION}

Multilayer films containing meso-tetraphenylporphyrins $\mathbf{1}$ and $\mathbf{2}$ with linkers $\mathbf{3}, \mathbf{4}$, and $\mathbf{5}$ have been fabricated on ITO using CuAAC and analyzed using GATR FT-IR, UV-vis spectroscopy and cyclic voltammetry. Infrared spectra of the multilayers suggest that the molecular subunits are reacting with the surface via
CuAAC, forming 1,4-triazole linked layers. The decrease in the intensity of the azide stretch in the IR spectrum after the addition of ethynyl-porphyrin layers as well as subsequent increase after the addition of the linker layer confirms that a CuAAC reaction is taking place as expected. When the azide stretch is monitored during multilayer growth, this oscillating trend is observed regardless of the linker used. However, only linker 5, which contains three azide groups, shows an overall increase in the amount of azide present in the film throughout multilayer growth. This suggests that two azides are reacting with the porphyrin layers sandwiching linker $\mathbf{5}$ and the third azide remains unreacted. An increase in intensity of IR features corresponding to porphyrin and linker peaks is observed with increasing number of layers. The average porphyrin tilt angle with respect to the surface can be calculated based on two of the IR features: $997 \mathrm{~cm}^{-1}$ in-plane porphyrin breathing mode and $797 \mathrm{~cm}^{-1}$ out of plane $\beta-\mathrm{H}$ deformation. Based on the comparison of the peak intensities in the IR spectrum of an isotropic sample of 1 in $\mathrm{KBr}$, the average porphyrin orientation for films grown with $\mathbf{1}$ and 3 or 5 was found to be $44^{\circ}$ for both samples. Polarized UV-vis spectra are in close agreement with this value ( 47 for multilayers made with linker $3,45^{\circ}$ for multilayers made with 5 ). Comparison of these angles to the range of possible angles determined through thickness and computational studies suggest that the porphyrins are attaching via a trans bonding motif in the multilayer structures. Results from GATR FT-IR indicated that there remained significant amounts of unreacted azide in the film created with $\mathbf{1}$ and 5. Attempts to access the remaining azide in the film were moderately successful. Films with porphyrin $\mathbf{2}$ and $\mathbf{5}$ were grown to investigate the incorporation of ethynylferrocene in the films with remaining azide. Cyclic voltammograms indicate that there 
is some incorporation of ethynyl ferrocene in the multilayer; however, the doping concentration was low. Future work will investigate the usefulness of smaller catalyst-ligand combinations in order to access the unreacted azide components in the film.

We have furthered the understanding of the molecular orientation and structure of porphyrin containing LbL molecular multilayer films grown using CuAAC. Work is underway to examine the usefulness of these films in photoelectrochemical applications, and a good understanding of their structure will assist in interpreting future results and tailoring these films to specific applications.

\section{EXPERIMENTAL SECTION}

Materials. Solvents, ACS reagent grade or better, were purchased from Sigma Aldrich or Fisher Scientific and used as received. Toluene was purged with nitrogen and dried over $4 \AA$ molecular sieves before use. Dichloromethane for electrochemistry was dried and purified by recirculating the nitrogen purged solvent through a solid-state column purification system (Vacuum Atmospheres Company, Hawthorne, CA) prior to use. Sodium ascorbate (Aldrich) and 4,4'-diazido-2,2' stilbenedisulfonic acid disodium salt tetrahydrate (3) (Fluka) were used as received. Tetrabutylammonium hexafluorophosphate (TBAP, Acros) was recrystallized twice from hot ethanol before use in electrochemical experiments. 11-Azidoundecyltrimethoxysilane, ${ }^{67} \mathrm{Zn}$ (II) 5,10,15,20-tetra(4-ethynylphenyl)porphyrin (1) was available from previous studies ${ }^{23,24}$ and was synthesized according to literature methods. ${ }^{68}$ Tris-(benzyltriazolylmethyl)amine (TBTA), ${ }^{69}$ 1,3,5-tris(azidomethyl)benzene (5), ${ }^{70}$ and 1,4 bix(azidomethyl)benzene $(4)^{71}$ were synthesized according to literature methods. ITO-coated glass slides were purchased from Delta Technologies (polished float glass, ITO coated one surface, $\left.R_{\mathrm{s}}=4-8 \Omega\right)$.

$\mathrm{Cu}$ (II) 5,10,15,20-tetra(4-ethynylphenyl)porphyrin (2) was synthesized similar to 1 except copper(II) acetate was added to a methanol/ chloroform solution of the free base porphyrin for 30 minutes to give $\mathrm{Cu}$ (II) 5,10,15,20-tetra(4-trimethylsilylethynylphenyl)porphyrin (calculated for $\mathrm{C}_{64} \mathrm{H}_{60} \mathrm{~N}_{4} \mathrm{CuSi}_{4}$ : 1059.3186; found: MALDI 1059.4, ESI-HR 1059.3193). Subsequent deprotection using 18-crown-6 in a solution of chloroform/ methanol/tetrahydrofuran for $2 \mathrm{~h}$ yielded $\mathrm{Cu}$ (II) 5,10,15,20-tetra(4-ethynylphenyl)porphyrin (2). Calcd for $\mathrm{C}_{64} \mathrm{H}_{28} \mathrm{~N}_{4} \mathrm{Cu}$, 771.1605; found, ESI-HR 771.1607. UV-vis (toluene): 421, $542 \mathrm{~nm}$.

Azido-SAM Formation on ITO. Prior to use, ITO-coated glass slides were sonicated in a dilute aqueous solution of Alconox for 5-10 min, washed with water, acetone, dichloromethane, methanol, and lastly water, then placed in concentrated sulfuric acid for at least $30 \mathrm{~min}$ to generate a high concentration of surface hydroxides. The slides were then rinsed with copious amounts of DI water, dried under a stream of nitrogen, and placed in a Schlenk flask at a pressure of 0.1 mtorr to remove residual water. Slides were submerged in a solution of approximately $1 \mathrm{mM} 11$-azidoundecyltrimethoxysilane in anhydrous toluene. The reaction vessel was then heated at $60-70^{\circ} \mathrm{C}$ overnight. After cooling to room temperature slides were removed and sonicated in toluene for 5 min, after which they were washed with acetone, dichloromethane, methanol, and water, and then dried in a stream of nitrogen. Slides were then placed in a vacuum oven at $75^{\circ} \mathrm{C}$ for $3 \mathrm{~h}$.

Multilayer Fabrication. Ethynyl-Porphyrin Layers. A solution of DMSO, containing $<2 \%$ water, consisting of $1.3 \mathrm{mM} 1,0.325 \mathrm{mM}$ $\mathrm{CuSO} 4,0.358 \mathrm{mM}$ TBTA, and $0.48 \mathrm{mM}$ sodium ascorbate was placed in contact with the SAM functionalized ITO side of a microscope slide. After $6 \mathrm{~min}$, the slide was washed with acetone, dichloromethane, ethanol, and water. Azidolinker layer: A DMSO solution, containing $<8 \%$ water, was used as described above consisting of $2.2 \mathrm{mM}$ of the selected azide functionalized linker molecule $(3,4$, or 5$), 4.4 \mathrm{mM} \mathrm{CuSO}_{4}$, $4.8 \mathrm{mM}$ TBTA, and $8.9 \mathrm{mM}$ sodium ascorbate. Sodium ascorbate stock solutions were replaced at regular intervals in order to guarantee activity.
For CuTPE porph 2, the reaction solution was made using tetrahydrofuran and dimethylsulfoxide as the solvent in order to improve solubility. No effort was made to remove copper from the film, although it is known that the films contain excess copper from the CuAAC catalyst. ${ }^{24,72}$

Infrared Spectroscopy. A Bio-Rad (Varian) Excalibur infrared spectrometer was used with a liquid nitrogen cooled MCT detector and VariGATR attenuated total reflectance accessory (Harrick Scientific) equipped with a single reflection hemispherical Ge crystal. Light was polarized with a Wire Grid Polarizer (Harrick Scientific). Thin film samples on ITO were pressed against the crystal surface using a high torque slip-clutch pressure applicator to ensure consistent pressure over all samples. The GATR accessory was purged with dried nitrogen during acquisition in order to maintain a constant atmosphere. The incident light was held at an angle of 60 degrees versus normal with the light polarized perpendicular to the surface. Samples were acquired with Varian Resolutions Pro software at a resolution of $4 \mathrm{~cm}^{-1}$ with 256 scans and ratioed to a freshly cleaned ITO slide that had been cleaned in a UVO cleaner (Bioforce ProCleaner plus) for at least 5 min. Resulting absorbance spectra were baseline corrected using multiple points void of major IR transitions. The baseline corrected spectra was used to determine the azide content in the films by integrating the azide stretching peak at $2100 \mathrm{~cm}^{-1}$. In order to determine the ratio between peaks at 997 and $797 \mathrm{~cm}^{-1}$, deconvoluted spectra were used to isolate the peaks of interest. $^{72,73}$ Quantification of these peaks by peak height or peak area resulted in similar calculated average molecular orientations. The isotropic IR spectrum of 1 was obtained by creating a homogeneous sample of 1 by grinding in $\mathrm{KBr}$ and pressing into a pellet with the same acquisition parameters described above.

Electronic Absorption Spectroscopy. UV-visible electronic absorption spectra were taken on a Perkin-Elmer Lamda 950 spectrometer with slides held normal to the incoming light beam in air. A background spectrum of the SAM functionalized slide was subtracted from each spectrum. Polarized absorbance spectra were taken with the slide positioned at a $45^{\circ}$ angle with respect to the incident light beam, and a background spectrum was subtracted for each angle at which a sample spectrum was taken. The incident light was polarized with a Glan-Taylor prism.

Electrochemistry. Electrochemical measurements were performed using a $\mathrm{CH}$ Instruments $440 \mathrm{~A}$ potentiostat in a three electrode configuration. The working area of the ITO functionalized electrode was defined by the cylindrically bored area of a Teflon cone pressed on the sample surface and filled with electrolyte. ${ }^{74}$ Multilayer thin film electrochemical experiments examining porphyrin oxidations were performed in dry DCM with $0.1 \mathrm{M} \mathrm{TBAP}$ as the electrolyte with a $\mathrm{Ag} / \mathrm{Ag}^{+}$pseudoreference electrode and a platinum wire counter electrode. Electrochemical measurements examining ferrocene concentration within the multilayers films were examined using $0.1 \mathrm{M}$ perchloric acid as the electrolyte, a $\mathrm{Ag} / \mathrm{AgCl}($ satd. $\mathrm{NaCl})$ reference electrode and a platinum wire counter electrode.

\section{ASSOCIATED CONTENT}

S Supporting Information. Cyclic voltammograms of 2 and $\mathbf{5}$ with ferrocene, polarized absorbance spectra of multilayers, schematic representation of multicomponent film assembly, scan rate dependence of ferrocene modified SAMs on ITO, and IR spectra for multicomponent films of $\mathbf{2}$ and $\mathbf{5}$ with ferrocene examining the azide stretching region. This material is available free of charge via the Internet at http://pubs.acs.org.

\section{AUTHOR INFORMATION}

\section{Corresponding Author}

*E-mail: dinolp@rpi.edu. 


\section{ACKNOWLEDGMENT}

We thank Dr. Ellen Miseo (Analytical Answers, Inc.) for assistance with IR analysis, interpretation, and instrumentation. This work was supported by Rensselaer Polytechnic Institute through new faculty start-up funds and by the National Science Foundation under Grant 0333314.

\section{REFERENCES}

(1) George, S. M.; Yoon, B.; Dameron, A. A. Acc. Chem. Res. 2009, $42,498-508$.

(2) van der Boom, M. E.; Marks, T. J. In Polymers for Microelectronics and Nanoelectronics; American Chemical Society: Washington, D.C., 2004; Vol. 874, pp 30-43.

(3) Cao, G.; Hong, H. G.; Mallouk, T. E. Acc. Chem. Res. 2002, 25, $420-427$.

(4) Lee, H.; Kepley, L. J.; Hong, H. G.; Akhter, S.; Mallouk, T. E. J. Phys. Chem. 2002, 92, 2597-2601.

(5) Yang, H. C.; Aoki, K.; Hong, H. G.; Sackett, D. D.; Arendt, M. F.; Yau, S. L.; Bell, C. M.; Mallouk, T. E. J. Am. Chem. Soc. 2002, $115,11855-11862$.

(6) Altman, M.; Shukla, A. D.; Zubkov, T.; Evmenenko, G.; Dutta, P.; van der Boom, M. E. J. Am. Chem. Soc. 2006, 128, 7374-7382.

(7) Altman, M.; Zenkina, O.; Evmenenko, G.; Dutta, P.; van der Boom, M. E. J. Am. Chem. Soc. 2008, 130, 5040-5041.

(8) Motiei, L.; Sassi, M.; Kaminker, R.; Evmenenko, G.; Dutta, P.; Iron, M. A.; van der Boom, M. E. Langmuir 2010, 27, 1319-1325.

(9) Motiei, L.; Lahav, M.; Gulino, A.; Iron, M. A.; van der Boom, M. E. J. Phys. Chem. B 2010, 114, 14283-14286.

(10) Altman, M.; Zenkina, O. V.; Ichiki, T.; Iron, M. A.; Evmenenko, G.; Dutta, P.; van der Boom, M. E. Chem. Mater. 2009, 21, 4676-4684.

(11) Li, D.; Ratner, M. A.; Marks, T. J.; Zhang, C.; Yang, J.; Wong, G. K. J. Am. Chem. Soc. 1990, 112, 7389-7390.

(12) Netzer, L.; Sagiv, J. J. Am. Chem. Soc. 1983, 105, 674-676.

(13) van der Boom, M. E.; Richter, A. G.; Malinsky, J. E.; Lee, P. A.; Armstrong, N. R.; Dutta, P.; Marks, T. J. Chem. Mater. 2000, 13, 15-17.

(14) van der Boom, M. E.; Zhu, P.; Evmenenko, G.; Malinsky, J. E.;

Lin, W.; Dutta, P.; Marks, T. J. Langmuir 2002, 18, 3704-3707.

(15) Zhu, P.; van der Boom, M. E.; Kang, H.; Evmenenko, G.; Dutta, P.; Marks, T. J. Chem. Mater. 2002, 14, 4982-4989.

(16) Jiao, J.; Anariba, F.; Tiznado, H.; Schmidt, I.; Lindsey, J. S.; Zaera, F.; Bocian, D. F. J. Am. Chem. Soc. 2006, 128, 6965-6974.

(17) Kohli, P.; Blanchard, G. J. Langmuir 2000, 16, 4655-4661.

(18) Li, D.; Buscher, C. T.; Swanson, B. I. Chem. Mater. 1994, 6, 803-810.

(19) Li, D.; Ratner, M. A.; Marks, T. J.; Zhang, C.; Yang, J.; Wong, G. K. J. Am. Chem. Soc. 2002, 112, 7389-7390.

(20) Li, Y.-h.; Wang, D.; Buriak, J. M. Langmuir 2010, 26, 1232-1238.

(21) Schmidt, I.; Jiao, J.; Thamyongkit, P.; Sharada, D. S.; Bocian, D. F.; Lindsey, J. S. J. Org. Chem. 2006, 71, 3033-3050.

(22) Decher, G. In Photonic and Optoelectronic Polymers; American Chemical Society: Washington, D.C., 1997; Vol. 672, pp 445-459.

(23) Palomaki, P. K. B.; Dinolfo, P. H. Langmuir 2010, 26, 9677-9685.

(24) Palomaki, P. K. B.; Krawicz, A.; Dinolfo, P. H. Langmuir 2011, 27, 4613-4622.

(25) Rostovtsev, V. V.; Green, L. G.; Fokin, V. V.; Sharpless, K. B. Angew. Chem. Int. Ed. 2002, 41, 2596-2599.

(26) Tornoe, C. W.; Christensen, C.; Meldal, M. J. Org. Chem. 2002, $67,3057-3064$.

(27) Collman, J. P.; Devaraj, N. K.; Chidsey, C. E. D. Langmuir 2004, 20, 1051-1053.

(28) Collman, J. P.; Devaraj, N. K.; Eberspacher, T. P. A; Chidsey, C. E. D. Langmuir 2006, 22, 2457-2464.

(29) Devaraj, N. K.; Collman, J. P. QSAR Comb. Sci. 2007, 26, $1253-1260$
(30) Devaraj, N. K.; Decreau, R. A.; Ebina, W.; Collman, J. P.; Chidsey, C. E. D. J. Phys. Chem. B 2006, 110, 15955-15962.

(31) Devaraj, N. K.; Dinolfo, P. H.; Chidsey, C. E. D.; Collman, J. P. J. Am. Chem. Soc. 2006, 128, 1794-1795.

(32) Ku, S.-Y.; Wong, K.-T.; Bard, A. J. J. Am. Chem. Soc. 2008, 130, $2392-2393$.

(33) Lummerstorfer, T.; Hoffmann, H. J. Phys. Chem. B 2004, 108, 3963-3966.

(34) Prakash, S.; Long, T. M.; Selby, J. C.; Moore, J. S.; Shannon, M. A. Anal. Chem. 2006, 79, 1661-1667.

(35) Bergbreiter, D. E.; Chance, B. S. Macromolecules 2007, 40, $5337-5343$.

(36) Such, G. K.; Quinn, J. F.; Quinn, A.; Tjipto, E.; Caruso, F. J. Am. Chem. Soc. 2006, 128, 9318-9319.

(37) Rydzek, G.; Thomann, J.-S.; Ameur, N. B.; Jierry, L.; Msini, P.; Ponche, A.; Contal, C.; Haitami, A. E. E.; Voegel, J.-C.; Senger, B.; Schaaf, P.; Frisch, B.; Boulmedais, F. Langmuir 2009, 26, 2816-2824.

(38) Vestberg, R.; Malkoch, M.; Kade, M.; Wu, P.; Fokin, V. V.; Sharpless, K. B.; Drockenmuller, E.; Hawker, C. J. J. Polym. Sci., Part A: Polym. Chem. 2007, 45, 2835-2846.

(39) Luo, L.; Frisbie, C. D. J. Am. Chem. Soc. 2010, 132, 8854-8855.

(40) Kadish, K.; Smith, K. M.; Guilard, R. The Porphyrin Handbook; Academic Press: New York, 1999.

(41) Jurowa, M.; Schuckmanb, A. E.; Batteasb, J. D.; Drain, C. M. Coord. Chem. Rev. 2010, 254, 2297-2310.

(42) Thamyongkit, P.; Yu, L.; Padmaja, K.; Jiao, J.; Bocian, D. F.; Lindsey, J. S. J. Org. Chem. 2006, 71, 1156-1171.

(43) Wasielewski, M. R. Chem. Rev. 1992, 92, 435-461.

(44) Abdelrazzaq, F. B.; Kwong, R. C.; Thompson, M. E. J. Am. Chem. Soc. 2002, 124, 4796-4803.

(45) Cho, Y.-J.; Ahn, T. K.; Song, H.; Kim, K. S.; Lee, C. Y.; Seo, W. S.; Lee, K.; Kim, S. K.; Kim, D.; Park, J. T. J. Am. Chem. Soc. 2005, $127,2380-2381$.

(46) Campbell, W. M.; Jolley, K. W.; Wagner, P.; Wagner, K.; Walsh, P. J.; Gordon, K. C.; Schmidt-Mende, L.; Nazeeruddin, M. K.; Wang, Q.; Grätzel, M.; David L., Officer J. Phys. Chem. C 2007, 111, 11760-11762.

(47) Odobel, F.; Blart, E.; Lagree, M.; Villieras, M.; Boujtita, H.; El Murr, N.; Caramori, S.; Bignozzi, A. C. J. Mater. Chem. 2003, 13, 502-510.

(48) Monti, D.; Nardis, S.; Stefanelli, M.; Paolesse, R.; Natale, C. D.; D’Amico, A. J. Sens. 2009, 2009, Article ID 856053, 10 pages.

(49) Li, X. Y.; Czernuszewicz, R. S.; Kincaid, J. R.; Spiro, T. G. J. Am. Chem. Soc. 1989, 111, 7012-7023.

(50) Li, X. Y.; Czernuszewicz, R. S.; Kincaid, J. R.; Su, Y. O.; Spiro, T. G. J. Phys. Chem. 1990, 94, 31-47.

(51) Colthup, N. B. J. Opt. Soc. Am. 1950, 40, 397-400.

(52) Tolstoy, V. P.; Chernyshova, I.; Skryshevsky, V. A. Handbook of Infrared Spectroscopy of Ultrathin Films; Wiley-Interscience: Hoboken, NJ, 2003.

(53) Due to the low molar absorptivity of ethynyl CC stretches near $2100 \mathrm{~cm}^{-1}$ compared to the azide stretch, it is assumed that the ethynyl stretch does not contribute significantly and the peak at $2100 \mathrm{~cm}^{-1}$ arises almost entirely from the azide stretch.

(54) Bohn, P. W. Annu. Rev. Phys. Chem. 1993, 44, 37-60.

(55) Bard, A. J.; Faulkner, L. R. In Electrochemical Methods: Fundamentals and Applications; 2nd ed.; John Wiley \& Sons, Inc.: New York, 2001.

(56) Rhodes, C. P.; Long, J. W.; Doescher, M. S.; Fontanella, J. J.; Rolison, D. R. J. Phys. Chem. B 2004, 108, 13079-13087.

(57) Wahab, A.; Bhattacharya, M.; Ghosh, S.; Samuelson, A. G.; Das, P. K. J. Phys. Chem. B 2008, 112, 2842-2847.

(58) Padmaja, K.; Wei, L.; Lindsey, J. S.; Bocian, D. F. J. Org. Chem. 2005, 70, 7972-7978.

(59) Chidsey, C. E. D.; Bertozzi, C. R.; Putvinski, T. M.; Mujsce, A. M. J. Am. Chem. Soc. 1990, 112, 4301-4306.

(60) Meldal, M.; TornA̧e, C. W. Chem. Rev. 2008, 108, 2952-3015.

(61) Yasseri, A. A.; Syomin, D.; Malinovskii, V. L.; Loewe, R. S.; Lindsey, J. S.; Zaera, F.; Bocian, D. F. J. Am. Chem. Soc. 2004, 126, 11944-11953. 
(62) Arnold, R.; Terfort, A.; Wöll, C. Langmuir 2001, 17, 4980-4989.

(63) Debe, M. K. J. Appl. Phys. 1984, 55, 3354-3366.

(64) Zhang, Z.; Hu, R.; Liu, Z. Langmuir 1999, 16, 1158-1162.

(65) Blasie, J. K.; Ereckinska, M.; Samuels, S.; Leigh, J. S. Biochim. Biophys. Acta 1978, 501, 33-52.

(66) Qian, D.-J.; Nakamura, C.; Ishida, T.; Wenk, S.-O.; Wakayama, T.; Takeda, S.; Miyake, J. Langmuir 2002, 18, 10237-10242.

(67) Fu, Y.-S.; Yu, S. J. Angew. Chem. Int. Ed. 2001, 40, 437-440.

(68) Onitsuka, K.; Kitajima, H.; Fujimoto, M.; Iuchi, A.; Takei, F.; Takahashi, S. Chem. Commun. 2002, 2576-2577.

(69) Chan, T. R.; Hilgraf, R.; Sharpless, K. B.; Fokin, V. V. Org. Lett. 2004, 6, 2853-2855.

(70) Song, Y.; Kohlmeir, E. K.; Meade, T. J. J. Am. Chem. Soc. 2008, 130, 6662-6663.

(71) Xu, L. Q.; Yao, F.; Fu, G.-D.; Shen, L. Macromolecules 2009, 42, 6385-6392.

(72) Cameron, D. G.; Moffatt, D. J. J. Test. Eval. 1984, 12, 78-85.

(73) Kauppinen, J. K.; Moffatt, D. J.; Mantsch, H. H.; Cameron, D. G. Appl. Spectrosc. 1981, 35, 271-276.

(74) Robinson, D. B.; Chidsey, C. E. D. J. Phys. Chem. B 2002, 106, 10706-10713. 